\title{
BASIC SCIENCE ARTICLE Hyperoxia causes miR199a-5p-mediated injury in the developing lung
}

\author{
Mohammad Afaque Alam ${ }^{1,2}$, Suhita Gayen nee Betal ${ }^{3}$, Zubair H. Aghai ${ }^{3}$ and Vineet Bhandari ${ }^{1}$
}

BACKGROUND: Hyperoxia-induced acute lung injury (HALI) is characterized by increased permeability and infiltration of inflammatory cells, impairment of alveolar development, and compromised lung function. Recent evidence has determined that microRNAs (miRs) are implicated in hyperoxia-induced lung injury, including bronchopulmonary dysplasia (BPD). However, the expression profile and functional role of miR199a-5p in developing lungs have not been reported.

METHODS: The present study was undertaken to explore the role of miR199a-5p in developing mice lungs and human neonates. We exposed neonatal mice for 7 days, mouse lung epithelial cells (MLE12), mouse lung endothelial cells (MLECs), and macrophages (RAW246.7), to hyperoxia at different time points.

RESULTS: Our results demonstrated enhanced miR199a-5p expression in hyperoxia-exposed mice lungs and cells, as well as in tracheal aspirates of infants developing BPD, with significant reduction in the expression of its target, caveolin- 1 . Next, we observed that miR199a-5p-mimic worsens HALI as evidenced by increased inflammatory cells, cytokines, and lung vascular markers. Conversely, miR199a-5p-inhibitor treatment attenuated HALI.

CONCLUSION: Thus, our findings suggest that miR199a-5p is a potential target for attenuating HALI pathophysiology in the developing lung. Moreover, miR199a-5p-inhibitor could be part of a novel therapeutic strategy for improving BPD in preterm neonates.

Pediatric Research (2019) 86:579-588; https://doi.org/10.1038/s41390-019-0524-3

\section{INTRODUCTION}

The developing lung undergoes well-orchestrated morphological changes during the early period of embryonic and fetal development. ${ }^{1}$ Hyperoxia (Hyp) can directly cause lung injury by the generation of reactive oxygen species (ROS). Exposure to oxygen in the preterm infant leads to Hyp-induced acute lung injury (HALI), which is characterized by increased pulmonary permeability and diffuse infiltration of various inflammatory cells and impairment of alveolar development. ${ }^{2}$ This is a major contributor to the pathogenesis of bronchopulmonary dysplasia (BPD) in human preterm neonates. ${ }^{3-5}$ Alveolar development undergoes a series of complex pathways and mechanisms involving microRNAs (miRs). miRs comprise a large family of evolutionarily conserved small non-coding RNAs ( 21-25 nucleotides) that regulate gene expression either by messenger RNA (mRNA) degradation or translational repression. ${ }^{6}$ miRs have been implicated in many vital roles in cell differentiation, development, proliferation, signaling, inflammation, and apoptosis. ${ }^{7}$ Recent work by our group has demonstrated the dynamic regulation of miR$34 a$ in developing lungs. ${ }^{7}$

Regardless of the identification of about 2680 novel human miRs and 1983 mouse miRs, only a few have been validated by published experiments. ${ }^{6-8}$ Hence, much remains to be investigated for their functional targets and clinical implications. It has been demonstrated that alteration of miR199a-5p expression has a role in the pathogenesis of cystic fibrosis (CF). ${ }^{9}$ miR199a-5p contributes to cell proliferation, motility, and angiogenesis by targeting the $3^{\prime}$-untranslated region (UTR) of caveolin-1 (Cav-1). ${ }^{9,10}$ Hence, we hypothesized that targeting miR199a-5p level may attenuate HALI in the newborn (NB) developing lung.

In the current study, we explored the regulation of miR199a-5p, its corresponding targets, and inflammatory markers following exposure of neonatal mice to $100 \% \mathrm{O}_{2}$ or room air (RA) for 7 postnatal (PN) days. Lungs of Hyp-exposed mice displayed histological changes consistent with HALI. ${ }^{11}$ Using gain(miR199a-5p-mimic) and loss- (miR199a-5p-inhibitor) of-function approaches in our in vivo model, we demonstrate the causal role of miR199a-5p in modulating lung protein vascular leak, inflammation, and architecture in HALI in the NB. Our results reveal that the dynamic regulation of miR199-5p plays a vital role in the pathophysiology of HALI that makes it a potential attractive target to attenuate HALI/BPD in the developing lung.

\section{MATERIALS AND METHODS}

All experimental wild-type (WT) mice of the C57BL/6 strain were purchased from The Jackson Laboratory (Bar Harbor, ME). Mice were housed and bred in Drexel University Animal Care Facilities. All mice were allowed ad libitum access to food and water. All animal procedures were performed in accordance with National Institutes of Health $(\mathrm{NIH})$ policies and approved by the Institutional Animal Care and Use Committee at Drexel University (Philadelphia, PA).

\footnotetext{
${ }^{1}$ Department of Pediatrics, Division of Neonatology, Drexel University College of Medicine, Philadelphia, PA, USA; ${ }^{2}$ Department of Neurosciences, Lewis Katz School of Medicine, Temple University, Philadelphia, PA, USA and ${ }^{3}$ Department of Pediatrics, Division of Neonatology, Thomas Jefferson University, Philadelphia, PA, USA Correspondence: Vineet Bhandari (vineet.bhandari@drexel.edu)
} 
Cell culture

Murine cell lines [mouse lung epithelial cells (MLE12), macrophage cell line (RAW264.70)] were purchased from the American Type Culture Collection (Manassas, VA) and cultured as previously described. ${ }^{12}$ Cells were maintained in Dulbecco's modified Eagle's medium (DMEM) supplemented with $10 \%$ fetal bovine serum (FBS) (HyClone) and supplemented with penicillin (100 U/ml)/ streptomycin $(100 \mu \mathrm{g} / \mathrm{ml}$; Invitrogen Life Technologies). Primary mouse lung endothelial cells (MLECs) and mouse endothelium cell medium (Cat. \# M1168) were purchased from Cell Biologics (Chicago, IL). Hyp conditions were achieved by placing $70-75 \%$ confluent cells for specified time points in $95 \% \mathrm{O}_{2} / 5 \% \mathrm{CO}_{2}$ at $37^{\circ} \mathrm{C}$ in a tightly sealed modular chamber, as described previously. ${ }^{7}$ For the $10-\mathrm{cm}$ plate, we used $10 \mathrm{ml}$ of culture media. For 6-well plates ( $35 \mathrm{~mm}$ ), we used $2 \mathrm{ml}$ of culture media. Cells were neither stirred nor rocked in the incubator. Control cells were cultured in a standard incubator $\left(21 \% \mathrm{O}_{2} / 5 \% \mathrm{CO}_{2}\right)$ at $37^{\circ} \mathrm{C}$. For MLECs, specific cell growth media were used. The buffer and glucose concentration in the $1 \times$ DMEM was as follows: $1 \mathrm{~g} / \mathrm{l}$ glucose, L-glutamine, and sodium pyruvate. Hyp-exposed cells were maintained under $5 \%$ $\mathrm{CO}_{2} / 95 \% \mathrm{O}_{2}$ for the duration of experiment. An oxygen analyzer (Pacifitech Industries, Los Angeles, CA) was used to confirm the $\mathrm{O}_{2}$ concentration in the incubator daily. Culture dishes were covered with a sterile, gas-permeable membrane (Diversified Biotech, Boston, MA) to allow equilibration of oxygen at the cellular surface. We exchanged the gas every $12 \mathrm{~h}$ in the sealed incubator. Four independent experiments were performed with three replicates in each experiment.

In vitro transfection of cells with miR199a-5p-mimic and miR199a$5 p$-inhibitor

Synthetic pre-miRs were purchased from Qiagen (miScript microRNA (miRNA) mimic and inhibitor, Qiagen, USA). Cells were seeded in $6-\mathrm{cm}$ dishes, grown to about $70 \%$ confluence, and then used for cell transfection according to the manufacturer's protocol, using miR199a-5p mimic $(50 \mathrm{nM})$ or miR199a-5p inhibitor $(100 \mathrm{nM})$. Cells were exposed to Hyp or RA for $0,4,16$, and $24 \mathrm{~h}$. Cells were harvested according to the experimental protocol. $^{7}$

\section{Exposure to Hyp}

NB mice, along with their mothers, were exposed to $100 \% \mathrm{O}_{2}$ in airtight polypropylene chamber (medium size), as previously described. ${ }^{13-16}$ Foster mothers were used to alternate dams every $24 \mathrm{~h}$ until the end of the experiment, to minimize toxicity to the adult mice, and to allow for adequate nutrition. Exposure to $100 \%$ $\mathrm{O}_{2}$ was initiated on PN1 (saccular stage of lung development) and continued till PN7 (early alveolar stage). During the period of Hyp, mice remained unrestrained and had free access to water and food and to a $12 \mathrm{~h}$ light-dark cycle. Oxygen levels were constantly monitored. Three independent experiments for a total of 8-12 neonatal mice pups in each group were conducted.

Human lung tracheal aspirates

Human lung tracheal aspirates (TAs) were obtained from premature infants being mechanically ventilated in the first PN week with an in-dwelling endotracheal tube. These infants had the final outcomes of having the diagnoses with or without BPD. BPD was defined as per the NIH consensus criteria. ${ }^{3}$ Collection and processing of the human lung samples was approved by the institutional review board of the Thomas Jefferson University Hospital. Selected clinical details (including $\mathrm{FiO}_{2}$ at the time of the TA collection) have been shown in Table 1 .

Intranasal delivery of miR199a-5p-mimic and miR199a-5p-inhibitor to NB mice pups

Mice pups from the same biological mother were divided in two groups (Hyp and RA). Each group was treated with
Table. 1. Clinical data for BPD and no BPD $(n=10)$

\begin{tabular}{llll}
\hline Parameters & All & BPD & No BPD \\
\hline Birth weight (g) & $761 \pm 206$ & $642 \pm 116$ & $881 \pm 251$ \\
Gestational age (weeks) & $25.8 \pm 2.0$ & $24.6 \pm 1.4$ & $27.0 \pm 2.2$ \\
Female sex (\%) & $7(70)$ & $3(60)$ & $4(80)$ \\
White race (\%) & $4(40)$ & $1(20)$ & $3(60)$ \\
Prenatal steroids (\%) & $10(100)$ & $5(100)$ & $5(100)$ \\
Fraction of inspired oxygen $\left(\mathrm{FiO}_{2}{ }^{\mathrm{a}}\right)$ & $0.35 \pm 0.05$ & $0.41 \pm 0.09$ & $0.28 \pm 0.02$ \\
Surfactant (\%) & $10(100)$ & $5(100)$ & $5(100)$ \\
Late-onset sepsis (\%) & $5(50)$ & $3(60)$ & $2(40)$ \\
Severe IVH (grade 3 or more) $(\%)$ & $6(60)$ & $4(80)$ & $2(40)$ \\
\hline
\end{tabular}

No significant difference between BPD and no BPD $(P=0.06$ for BW and GA)

$B P D$ bronchopulmonary dysplasia, $I V H$ intraventricular hemorrhage, $B W$ body weight, $G A$ gestational age

alf room air values were excluded from both groups, then BPD vs. no BPD values were $0.54 \pm 0.05$ vs. $0.3 \pm 0.0, P=0.0016$

Data expressed as mean \pm SEM

miR199a-5p-mimic, miR199a-5p-inhibitor, and scrambled miR at PN2 and at PN4 at a concentration of $20 \mu \mathrm{M}$, as previously described $^{7}$, and then sacrificed on PN7.

Quantitative real-time polymerase chain reaction (miR and mRNA expression analysis)

The endogenous miR199a-5p and mRNA expression levels of Cav-1, Toll-like receptor 4 (TLR4), tumor necrosis factor- $a$ (TNF-a), interleukin-6 (IL-6), and E-selectin were measured by quantitative real-time polymerase chain reaction (PCR). Total RNA was extracted from epithelial or MLE12, endothelial or MLECs, and macrophage or RAW264.7 cell lines, lungs, and human TA pellets using miRNeasy mini kit (Qiagen, Valencia, CA). RNA concentration was measured in NanoDrop 2000 (Thermo Scientific ${ }^{\mathrm{TM}}$ ) and 260/ 280 ratio was $>2.0$. The complementary DNAs (cDNAs) were synthesized by a reverse transcription kit according to the manufacturer's instructions (Applied Biosystem). The StepOnePlus $^{\mathrm{TM}}$ platform (Applied Biosystems) was used for all PCR, done in triplicate using miScript primer assay (Qiagen). Changes in expression were calculated by the change in threshold $(\Delta \Delta C T)$ and the expression levels of the miRs were normalized to those of RNU6 small nuclear RNA and the expression levels of the target genes were normalized to those of hypoxanthine-guanine phosphoribosyltransferase. The thermal profile for PCR was $95^{\circ} \mathrm{C}$ for $5 \mathrm{~min}$, followed by 40 cycles of $15 \mathrm{~s}$ at $94^{\circ} \mathrm{C}$, with $30 \mathrm{~s}$ annealing followed by $45 \mathrm{~s}$ extension at $72{ }^{\circ} \mathrm{C}$. The specific primers were designed as shown in Table 2.

Lung morphometric analysis

Lungs were inflated and fixed in $4 \%$ phosphate-buffered formalin, immersed in fixative overnight, paraffin embedded, sectioned at $5 \mu \mathrm{m}$, and stained with hematoxylin and eosin (H\&E). Five non-overlapping photomicrographs in different sections were captured at $\times 10$ magnification. Alveolar size was estimated from the mean chord length of the airspace and septal thickness, as described previously. ${ }^{15,16}$ Alveolar area, numbers of alveoli, branches, and junctions were calculated using Analyze Skeleton program from ImageJ. This plugin tags all pixel/voxels in a skeleton image and then measures the surface area of alveoli and counts the number of alveoli, and all its junctions and branches, as previously described. ${ }^{17}$

Please see Supplemental Methods for additional rodent model of HALI, bronchoalveolar lavage fluid (BALF) and lung 
Table. 2. Primers used for quantitative real-time PCR

\begin{tabular}{lll}
\hline Genes & Forward primer $\left(5^{\prime} \rightarrow 3^{\prime}\right)$ & Reverse primer $\left(5^{\prime} \rightarrow 3^{\prime}\right)$ \\
\hline Cav-1 & CTA CAA GCC CAA CAA CAA GGC & AGG AAG CTC TTG ATG CAC GGT \\
$T L R 4$ & AGT ATC GAG AGG CTC AGG TAT AG & TAC AGG ATG CAG GAC AAG TAA TC \\
IL-6 6 & CCT CTG GTC TTC TGG AGT ACC & GCT GGA GTC ACA GAA GGA GT \\
TNF- $a$ & ATG AGC ACA GAA AGC ATG A & ACC ACG CTC TTC TGT CTA CT \\
E-selectin & AAC TGC GAG AAG AAC GGA TAG & GCG CTA GAC TGG AGA AAC TT \\
\hline
\end{tabular}
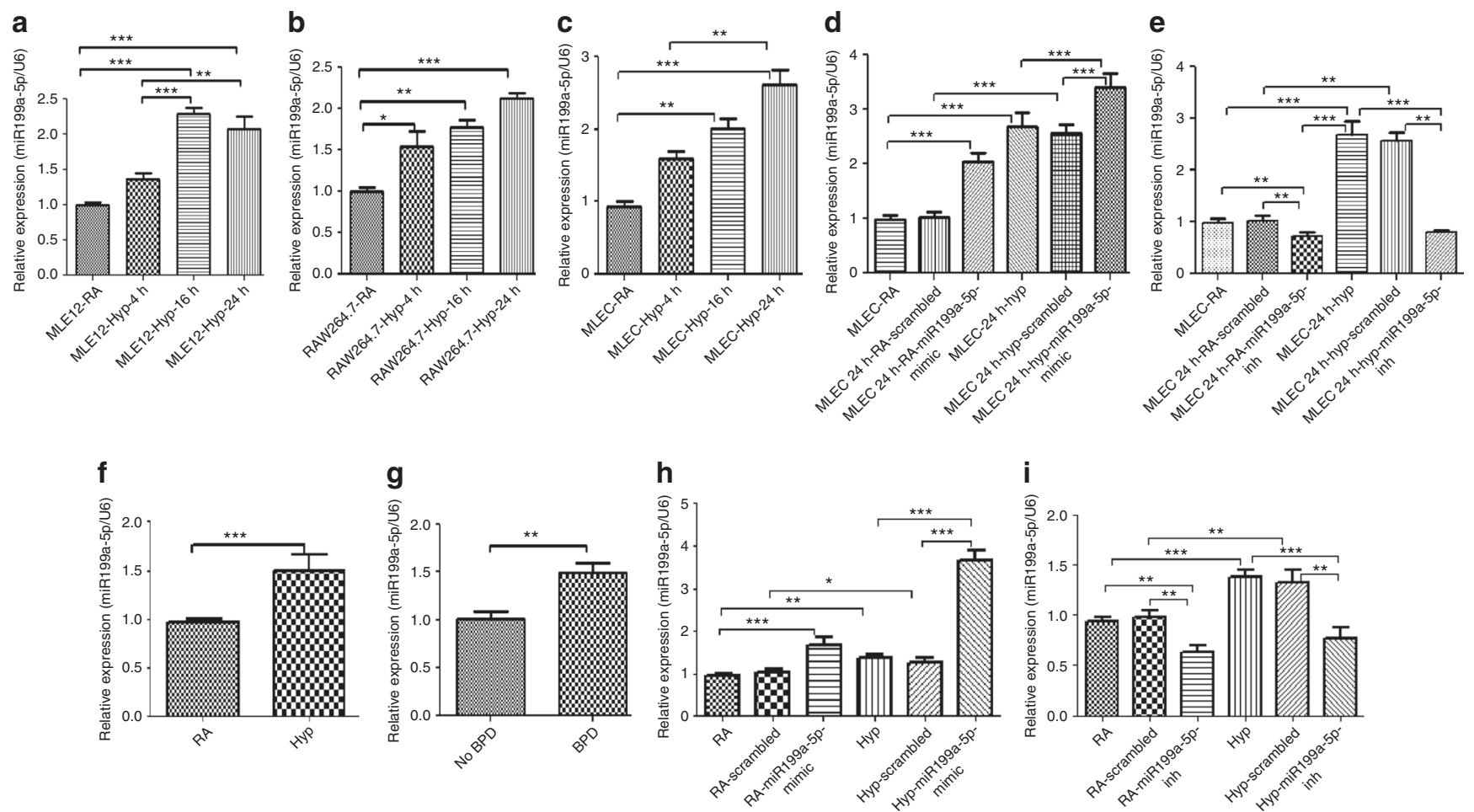

Fig. 1 Hyperoxia (Hyp) induces miR199a-5p expression in vitro and in vivo. miR199a-5p expression levels were measured by real-time polymerase chain reaction (PCR) in room air (RA) and Hyp-exposed MLE12, RAW264.7 cells, and mouse lung endothelial cells (MLECs) at 0, 4, 16 , and $24 \mathrm{hs}(\mathbf{a}-\mathbf{c})$, and in MLECs treated with miR199a-5p-mimic and miR199a-5p-inhibitor (along with scrambled controls) at $24 \mathrm{~h}$, after exposure to RA or hyperoxia (Hyp) (d, e). miR199a-5p expression levels were measured by real-time PCR in newborn (NB) mice (f) lungs exposed to RA and Hyp for 7 days, and in human tracheal aspirate samples (g). miR199a-5p expression levels were measured in miR199a-5pmimic and miR199a-5p-inhibitor (along with scrambled controls) administered mice lungs exposed to RA and Hyp for 7 days (h, i). Results expressed as the mean \pm SEM of data obtained. For the cell culture experiments, each graph represents data from four independent experiments with three replicates in each experiment. For the animal studies, each graph represents 8-12 animals in each group. BPD: bronchopulmonary dysplasia. ${ }^{*} P<0.05,{ }^{* *} P<0.01$, and ${ }^{* * *} P<0.001$

tissue collection, BALF neutrophil and macrophage counts, and immunoblotting.

\section{Statistical analyses}

Data are presented as mean \pm SEM. Groups were compared with the Student's two-tailed unpaired $t$ test or one-way analysis of variance, followed by Tukey's post hoc test for multigroup comparisons using GraphPad Prism 7.0 (GraphPad Software, Inc., San Diego, CA). $P<0.05$ was considered significant.

\section{RESULTS}

HALI upregulates miR199a-5p in developing lungs

To assess which cell types of the lung could be responsible for the elevated expression of miR199a-5p in Hyp conditions, we first evaluated the expression of miR199a-5p in mouse lung epithelial cells (MLE12; Fig. 1a), macrophage (RAW264.7; Fig. 1b), and MLECs (Fig. 1C). It was significantly increased in all three cell types (Fig. 1a-c). Although the miR199a-5p expression was decreased in MLE12 cells at the $24 \mathrm{~h}$ time point, it was still significantly increased over baseline values (Fig. 1a). This decrease could be due to increased cell death at that time point. Next, we examined the effect of miR199a-5p-mimic and miR199a-5p-inhibitor on MLEC (in RA and Hyp conditions), and it was found to be significantly increased and decreased (Fig. 1d, e), respectively. After the cell line studies, we extended our experiments to determine the contribution of miR199a-5p in HALI mice lungs of NB WT mice and NB lipopolysaccharide (LPS) WT rat exposed to Hyp and noted that miR199a-5p expression was significantly increased in the HALI models compared to RA (Fig. If and 

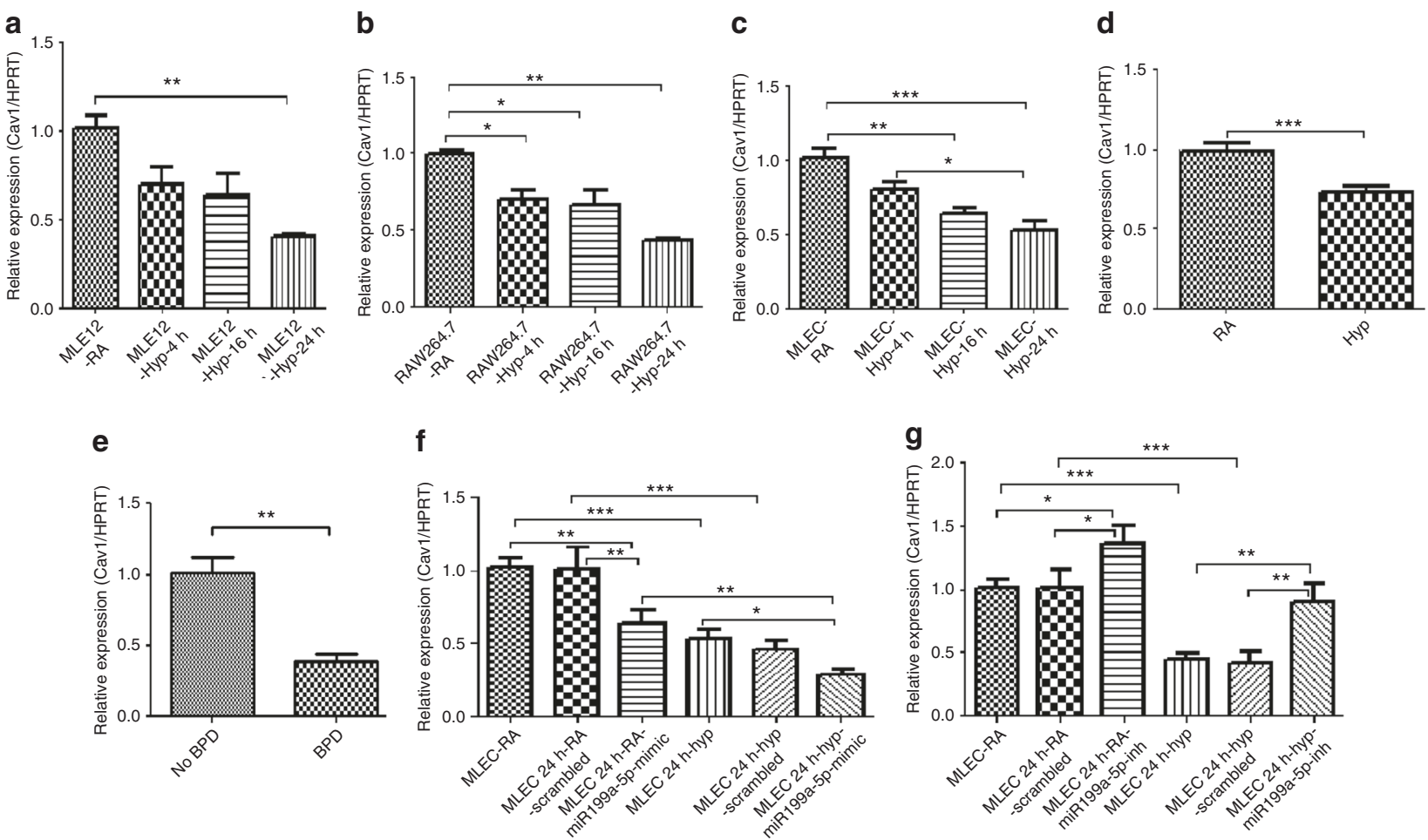

Fig. 2 Hyperoxia (Hyp) attenuates caveolin-1 expression in vitro and in vivo. Caveolin-1 (Cav-1) expression was measured by real-time PCR in the MLE12, RAW264.7 cells, and mouse lung endothelial cells (MLECs) at 0, 4, 16, and $24 \mathrm{~h}$ (a-c), and in newborn (NB) mice lungs exposed to room air (RA) and Hyp for 7 days, and in human tracheal aspirate (TA) samples (d, e). Cav-1 messenger RNA (mRNA) was measured after administering miR199a-5p-mimic (f) and miR199a-5p-inhibitor (g) (along with scrambled controls) in MLECs at $24 \mathrm{~h}$, after exposure to RA or Hyp; differences are reported as fold change. For the cell culture experiments, each graph represents data from four independent experiments with three replicates in each experiment. For the animal studies, each graph represents 8-12 animals in each group. LPS: lipopolysaccharide; BPD: bronchopulmonary dysplasia. ${ }^{*} P<0.05$, ${ }^{* *} P<0.01$, and ${ }^{* * *} P<0.001$

Supplemental Fig. S1a). In the human TA samples collected in the first PN week, infants who went on to develop BPD exhibited significantly increased miR199a-5p expression than the controls (Fig. 1g). Next, we gave miR199a-5p-mimic and miR199a-5pinhibitor intranasally to NB mice during Hyp and RA. Our results showed that miR199a-5p expression increased many folds in mimic and decreased in inhibitor administered mice pups respectively (Fig. $1 \mathrm{~h}$, i).

Thus, taken together, our data suggest that miR199a-5p expression is increased upon Hyp exposure in developing lungs and this appears to be localized at least (as these three were the only ones tested) to epithelial, endothelial, and macrophage cell types, as noted above.

Hyp diminished Cav-1 in cells (in vitro) and developing lungs (in vivo)

Cav-1 has been previously shown to be a direct target of miR199a$5 p$ in lung tissue., ${ }^{9,10}$ Hence, we explored the expression of this target gene for miR199a-5p. It was found that Cav-1 was significantly reduced in Hyp-exposed cells (epithelial, macrophages, and endothelial) (Fig. 2a-c) and in lung tissues of HALI mice and rats, as well as in TA of BPD infants (Fig. 2d, e, Supplemental Fig. S1b). Target scan predicts position of miR199a$5 p$ target site in CAV1 $3^{\prime}$-UTR and sequence alignment of miR199a-5p and the CAV1 3'-UTR from various species are shown. The "seed" region with a conserved WC match to the eighth nucleotide of the miRNA is highlighted (Supplemental Fig. S1c). Our results from MLEC experiments exhibited that Cav-1 mRNA expression was decreased with miR199a-5p-mimic and increased with miR199a-5p-inhibitor groups, respectively (Fig. 2f, g).
miR199a-5p-mimic enhanced inflammatory markers both in vitro and in vivo in HALI

Next, we examined mRNA expression for inflammatory cytokines and signaling molecules IL-6, TNF-a, TLR4, and E-selectin and found them to be significantly increased in Hyp-exposed cells and further enhanced in miR199a-5p-mimic groups (Fig. 3a-d). Likewise, we also noted that all were significantly increased in Hypexposed mice as well as those treated with miR199a-5p-mimic, compared to the respective RA controls (Fig. $3 \mathrm{e}-\mathrm{h}$ ).

Overexpression of miR199a-5p in HALI worsens lung vascular leak, inflammation, and architecture

To determine the contribution of overexpressed miR199a-5p in mice during HALI, we administered miR199a-5p-mimic to both RA and Hyp groups intranasally at PN2 and PN4 and sacrificed them at PN7. Our results demonstrated that BALF total cell counts were significantly increased during Hyp and they were further increased in the miR199a-5p-mimic administered mice (Fig. 4a). Simultaneously, we observed that the miR199a-5p-mimic worsened lung vascular leak and inflammation as noted by significantly increased protein leak (Fig. 4b), neutrophil, and macrophage influx (Fig. 4c, d). Moreover, we also noted that IL-6 levels were significantly increased, as measured by enzyme-linked immunosorbent assay (Fig. 4e). Furthermore, in the in vivo model, Cav-1 was decreased in Hyp and it further decreased significantly at both mRNA (Fig. 4f) and protein levels when given miR199a-5p-mimic during Hyp (Fig. 4g, i). It has been reported that miR199a-5p is involved in the Akt pathway, ${ }^{9}$ and so we measured Akt and post-translational phosphorylation of Akt (pAkt) and found significantly decreased expression in Hyp along with miR199a-5p-mimic groups (Fig. 4h, i). 

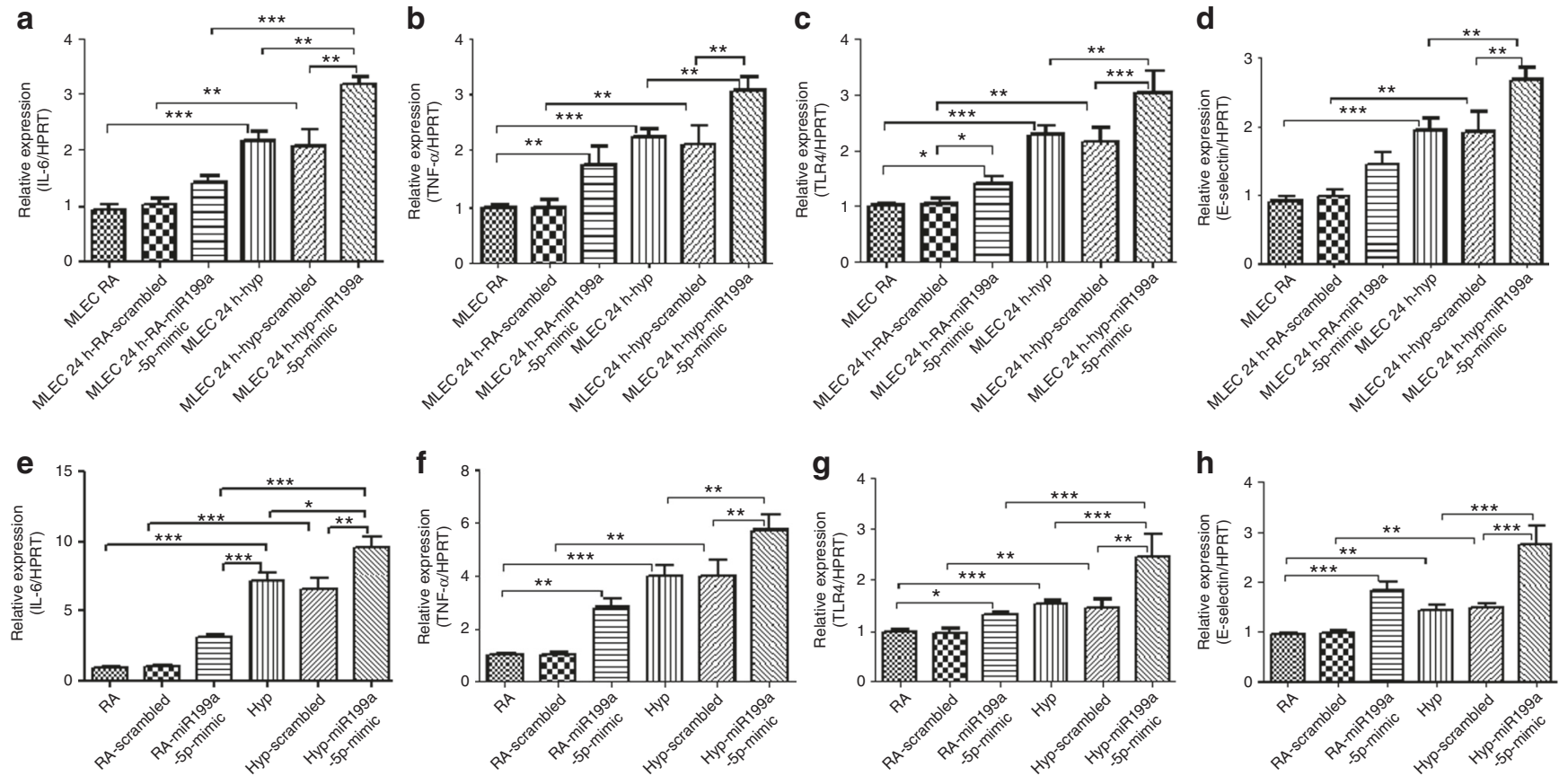

Fig. 3 miR199a-5p-mimic enhanced inflammatory markers both in vitro and in vivo in hyperoxia (Hyp)-induced acute lung injury (HALI). Bar graphs of interleukin-6 (IL-6), tumor necrosis factor- $\alpha$ (TNF- $\alpha$ ), Toll-like receptor 4 (TLR4), and E-selectin messenger RNA (mRNA) in mouse lung endothelial cells (MLECs) treated with miR199a-5p-mimic (along with scrambled controls) at $24 \mathrm{~h}$, after exposure to room air (RA) or Hyp (a-d). Bar graphs of IL-6, TNF- $\alpha$, TLR4, and E-selectin mRNA expression in lungs of newborn (NB) mice treated with miR199a-5p-mimic (along with scrambled controls), after exposure to RA or Hyp for 7 postnatal (PN) days (e-h). For the cell culture experiments, each graph represents data from four independent experiments with three replicates in each experiment. For the animal studies, each graph represents 8-12 animals in each group. ${ }^{*} P<0.05,{ }^{*} P<0.01$, and ${ }^{* * *} P<0.001$
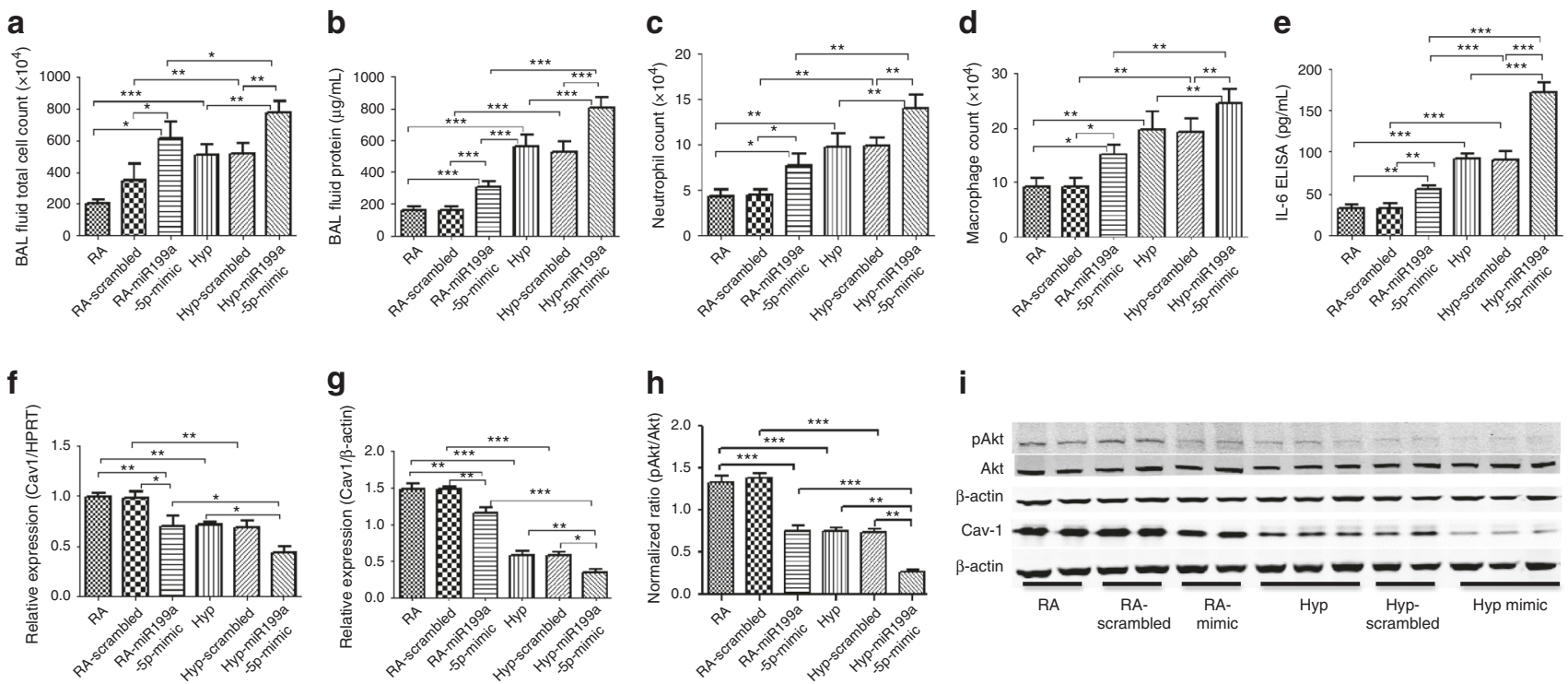

i

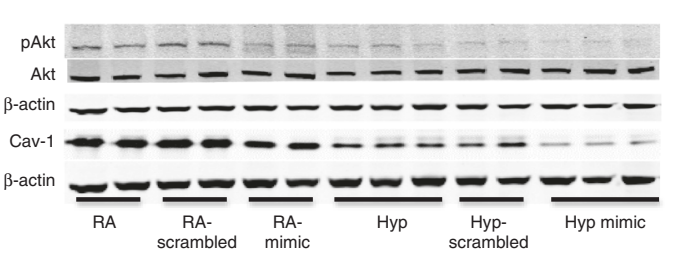

Fig. 4 miR199a-5p overexpression worsens pulmonary phenotype in hyperoxia (Hyp)-induced acute lung injury (HALI). Bar graphs of bronchoalveolar lavage (BAL) fluid total cell counts (a), protein leak (b), differential (neutrophils and macrophages) cell counts (c, d), and interleukin-6 (IL-6) levels (e) in miR199a-5p-mimic (along with scrambled control) administered mice lungs exposed to room air (RA) and Hyp for 7 days. Caveolin-1 (Cav-1) messenger RNA (mRNA) (f), protein (g, i), and ratio of phosphorylation of Akt (pAkt)/Akt protein (h, i) levels were measured in lung lysates of miR199a-5p-mimic (along with scrambled control) administered mice lungs exposed to RA and Hyp for 7 days. Values are means \pm SEM. For the cell culture experiments, each graph represents data from four independent experiments with three replicates in each experiment. For the animal studies, each graph represents 8-12 animals in each group. ${ }^{*} P<0.05$, ${ }^{* *} P<0.01$, and ${ }^{* * * P}<0.001$

In addition, lung architecture (Fig. 5a-f) significantly worsened in HALI and further deteriorated in the miR199a-5p-mimic groups during Hyp, as evidenced by lung morphometric analyses such as dysregulated chord length, alveolar areas, alveolar counts, septal thickness, number of branches, and junctions, as compared to their RA counterparts (Fig. $5 \mathrm{~g}-\mathrm{I}$ ).

We also explored the effect of miR199a-5p-mimic on apoptosis. As expected, apoptotic cells were more in Hyp and in miR199a-5p- 


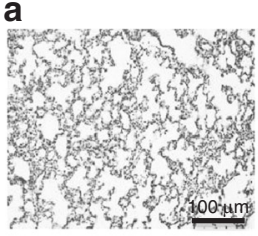

RA

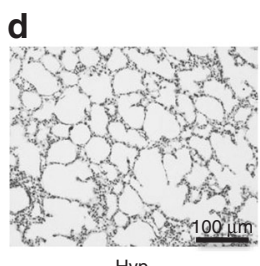

Hyp

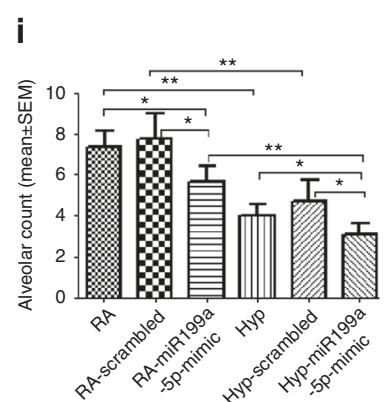

b

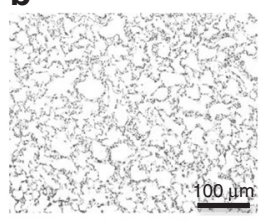

RA scrambled

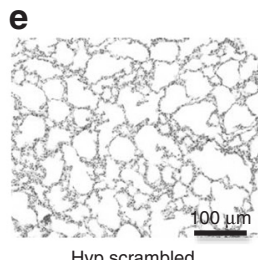

j

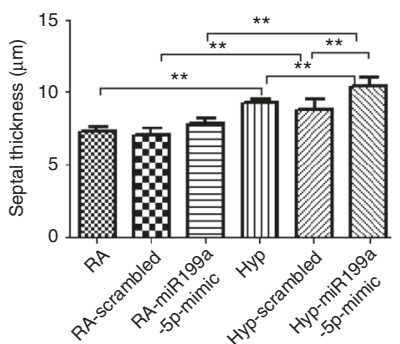

RA miR199a-5p-mimic
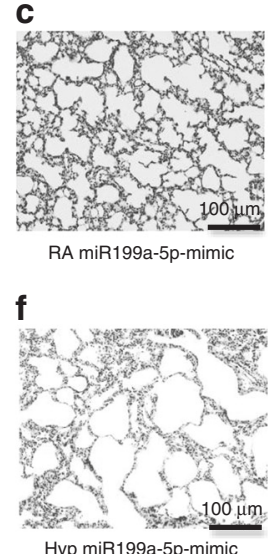

$\mathbf{k}$

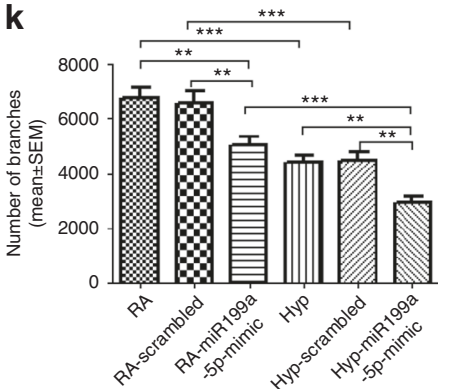

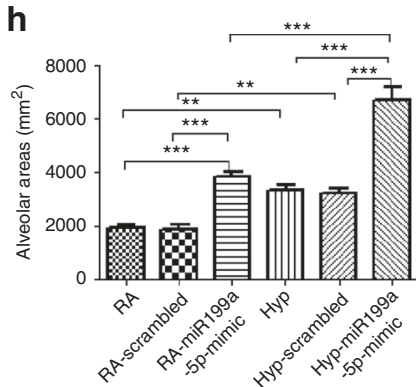

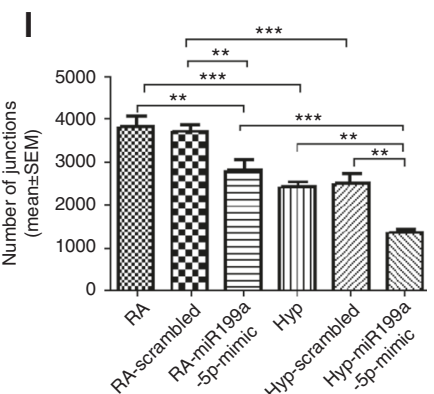

Fig. 5 miR199a-5p overexpression worsens lung architecture in hyperoxia (Hyp)-induced acute lung injury (HALI). Representative images of lung histology (hematoxylin and eosin (H\&E) stain) of newborn (NB) wild-type mice treated with miR199a-5p-mimic (20 $\mu$ M; PN2 and PN4 intranasally), along with appropriate controls, after room air (RA) or Hyp exposure for 7 postnatal (PN) days (a-f). Morphometry assessed as chord length, alveolar area, alveolar count, septal thickness, and number of branches and junctions (g-I), as analyzed by the ImageJ software. Values are means \pm SEM of $8-12$ animals in each group. ${ }^{*} P<0.05,{ }^{* *} P<0.01$, and ${ }^{* * *} P<0.001$

mimic in phase-contrast microscopy (Supplemental Fig. S2). Western blot of cleaved caspase-3 protein expression was found to be significantly increased, while Bcl2 was significantly decreased in miR199a-5p-mimic-treated mice (Supplemental Fig. S3a, b).

Taken together, our data suggest that miR199a-5p-mimic significantly worsens lung injury in Hyp-exposed lungs, and this is associated with a decrease in miR199a-5p downstream target, Cav-1, and an increase in proinflammatory signaling molecules, and increased cell death.

Inhibition of miR199a-5p expression in HALI attenuates lung vascular leak, inflammation, and architecture

In order to see the effect of miR199a-5p-inhibitor on HALI in NB mice, we observed that the miR199a-5p-inhibitor significantly improved lung vascular leak and inflammation, as demonstrated by decreased BALF total cell counts, protein leak, neutrophil influx, and macrophages (Fig. 6a-d). The above findings were further supported by significantly decreased IL-6 levels (Fig. 6e). Next, we performed RT-PCR and immunoblot to examine Cav-1 mRNA and protein expression and found those to be significantly increased at both mRNA (Fig. 6f) and protein levels in the miR199a-5pinhibitor-treated group (Fig. $6 \mathrm{~g}$, i) and pAkt/Akt protein expression was significantly increased in the miR199a-5p-inhibitor group (Fig. $6 \mathrm{~h}, \mathrm{i})$. Additionally, we investigated and found proinflammatory cytokines and signaling molecules (IL-6, TNF-a, TLR4, and E-selectin) to be decreased in MLEC miR199a-5p-inhibitor group (Fig. 7a-d). Similar results were noted in mice lung tissues compared to their respective controls (Fig. 7e-h). In addition, histological study of the lung architecture (Fig. 8a-f) was significantly improved as evidenced by improved chord length, alveolar areas, alveolar counts, septal thickness, and number of branches, as compared to their respective controls (Fig. 8g-l).
Apoptosis was measured as cleaved caspase 3 protein expression by Western blot and found to be significantly decreased, while $\mathrm{BCl} 2$ was significantly increased in miR199a-5p-inhibitor-treated mice (Supplemental Fig. S4a, b).

Taken together, our results suggest that the miR199a-5pinhibitor significantly attenuates lung injury in Hyp-exposed groups, is associated with an enhancement in miR199a-5p downstream target, Cav-1, and a decrease in proinflammatory signaling molecules, and improved cell survival.

\section{DISCUSSION}

The induction of miR199a-5p expression in lungs of NB mice, NB rat model of antenatal LPS combined with postnatal Hyp, and human BPD hints at the translational significance of our data. The in vitro findings from the lung epithelial, endothelial, and macrophage cells were corroborated with the in vivo data. Recently, Zhang et al. ${ }^{9}$ have reported a high miR199a-5p levels in human and murine CF macrophages and murine CF lungs contributing to lung "hyper-inflammation." Studies on patients with chronic obstructive pulmonary disease (COPD) and fibrotic lung diseases, especially idiopathic pulmonary fibrosis have shown to be associated with increased miR199a-5p expression. ${ }^{10,18,19}$ These data signify that aberrant expression of miR199a-5p may be involved in lung pathophysiology.

Scientific literatures have implicated the role of miRs in lung diseases such as $\mathrm{COPD}^{20}$ and BPD. ${ }^{7,21}$ Of note, several researchers have reported involvement of miRs in inflammation and immune response of various cell types of lung origin., ${ }^{7,22} \mathrm{~A}$ recent publication reported a decreased expression of miR-150 in the epithelium in neonatal mice lungs upon Hyp exposure. ${ }^{22}$ Investigators have implicated the miR-200 family in the 


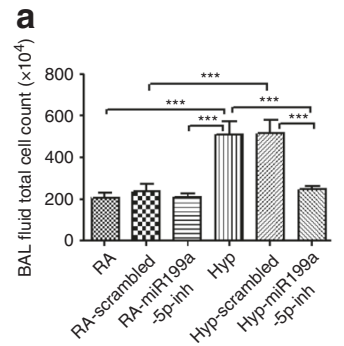

\section{b}

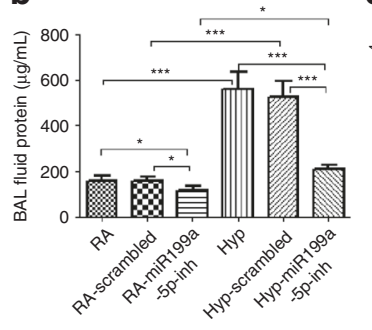

g

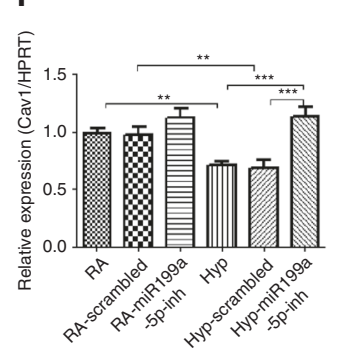

c

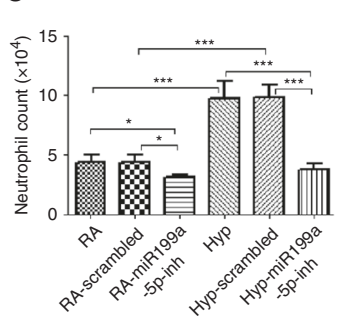

h

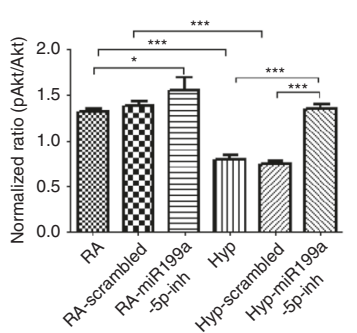

d
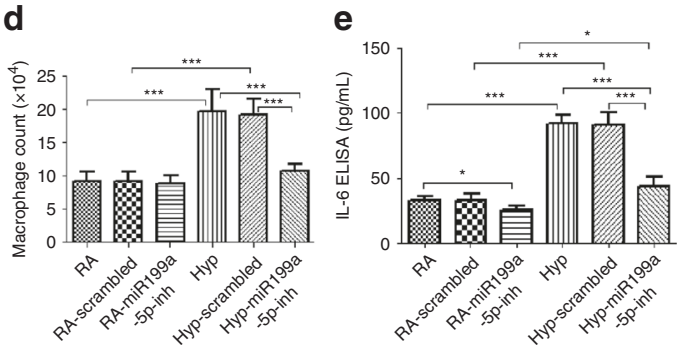

i

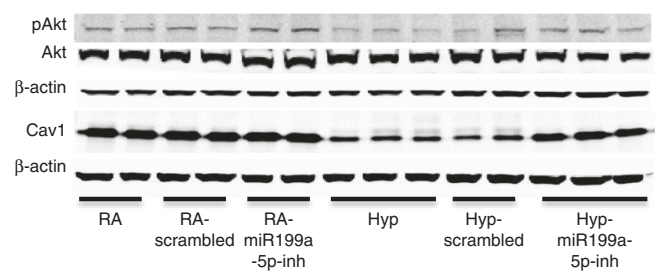

Fig. 6 miR199a-5p-inhibitor results in attenuation of pulmonary phenotype in hyperoxia (Hyp)-induced acute lung injury (HALI). Bar graphs of bronchoalveolar lavage (BAL) fluid total cell counts (a), protein leak (b), differential (neutrophils and macrophages) cell counts (c, d), and interleukin-6 (IL-6) levels (e) in miR199a-5p-inhibitor (along with scrambled control) administered mice lungs exposed to room air (RA) and Hyp for 7 days. Caveolin-1 (Cav-1) messenger RNA (mRNA) (f) and protein (g, i) and ratio of phosphorylation of Akt (pAkt)/Akt protein (h, i) expression in the miR199a-5p-inhibitor (along with scrambled control) treated groups. Values are means \pm SEM of 8-12 animals in each group. ${ }^{*} P<0.05,{ }^{* *} P<0.01$, and ${ }^{* * *} P<0.001$
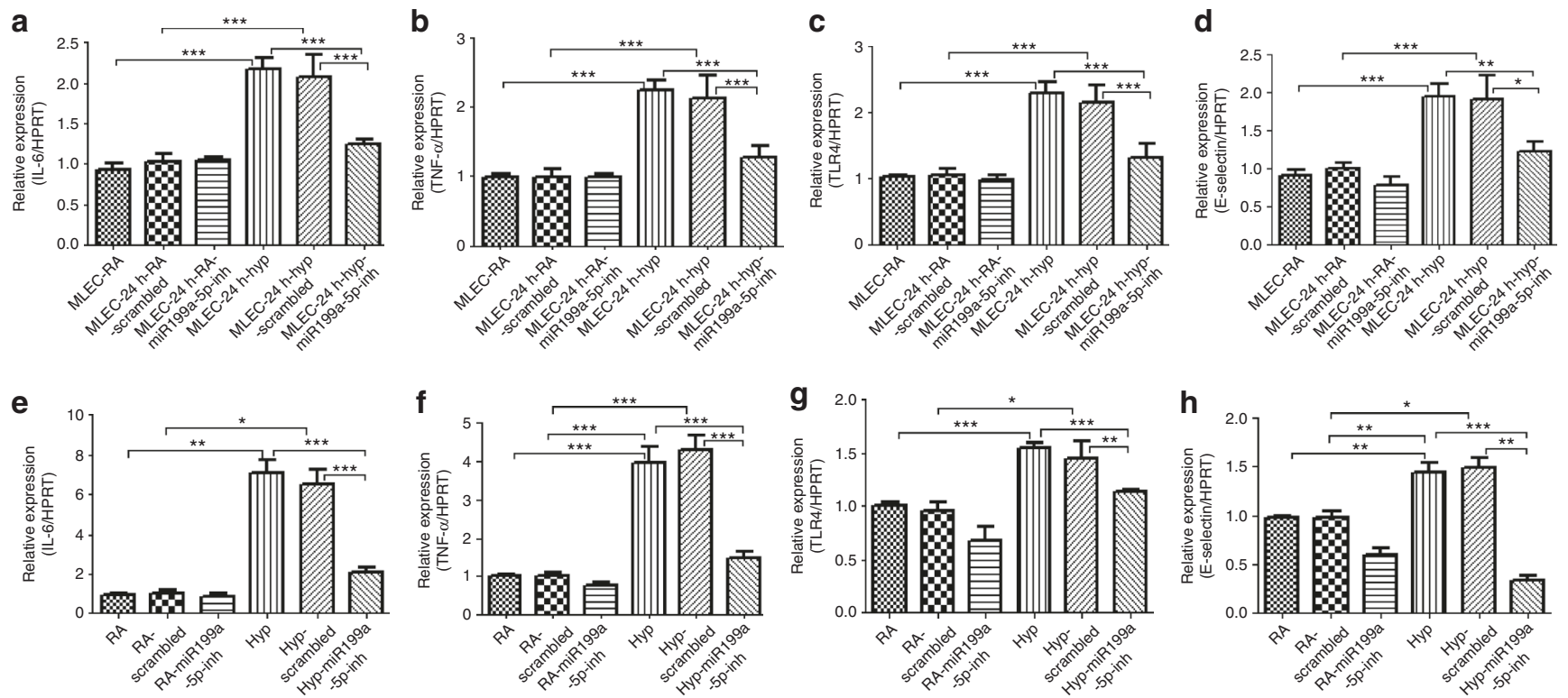

Fig. 7 miR199a-5p-inhibitor decreased inflammatory markers both in vitro and in vivo in hyperoxia (Hyp)-induced acute lung injury (HALI). Bar graphs of interleukin-6 (IL-6), tumor necrosis factor- $\alpha$ (TNF- $\alpha$ ), toll-like receptor (TLR4), and E-selectin messenger RNA (mRNA) in mouse lung endothelial cells (MLECs) treated with miR199a-5p-inhibitor (along with scrambled controls) at $24 \mathrm{~h}$, after exposure to room air (RA) or Hyp (a-d). Bar graphs of IL-6, TNF- $\alpha$, TLR4, and E-selectin mRNA expression in lungs of newborn (NB) mice treated with miR199a-5p-inhibitor (along with scrambled controls), after exposure to RA or Hyp for 7 postnatal (PN) days (e-h). For the cell culture experiments, each graph represents data from four independent experiments with three replicates in each experiment. For the animal studies, each graph represents 8-12 animals in each group. ${ }^{*} P<0.05,{ }^{* *} P<0.01$, and ${ }^{* *} P<0.001$

developmental regulation of Type II cell differentiation and function in human fetal lungs. ${ }^{23}$ We have described the role of miR-34a, ${ }^{7}$ while other investigators have implicated miR-489, ${ }^{24}$ miR-196a, ${ }^{25}$ and miR-29b ${ }^{8}$ as having a role in alveolar development and/or response to Hyp-induced injury in the developing lung.
Cav-1 is an important $22-\mathrm{kDa}$ transmembrane scaffolding protein abundantly present in epithelial, endothelial, and smooth muscle cells, as well as fibroblasts, neutrophils, and macrophages. It governs vital cellular functions such as membrane trafficking, cell proliferation and differentiation, apoptosis, and transcytosis through multiple signaling pathways. ${ }^{26,27}$ We found 


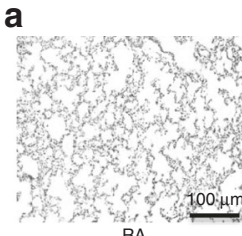

b

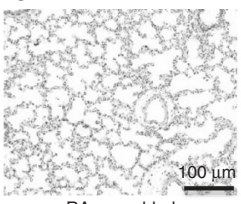

d
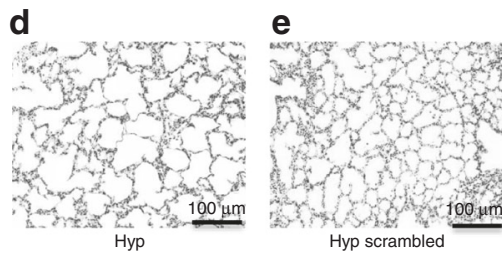
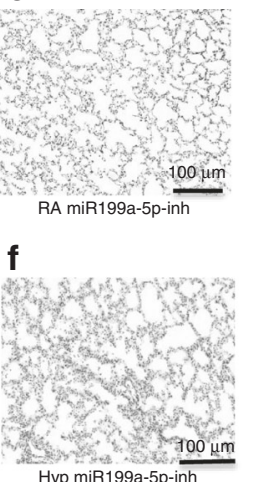

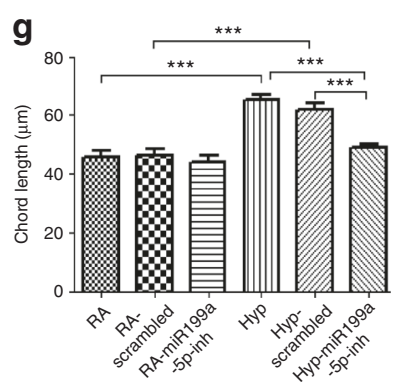

h

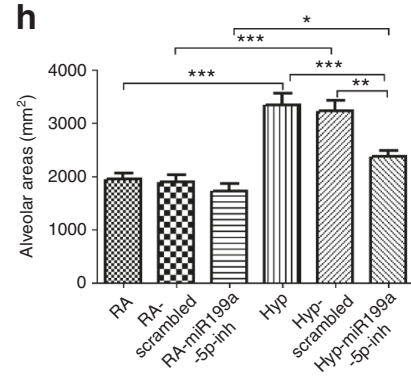

i

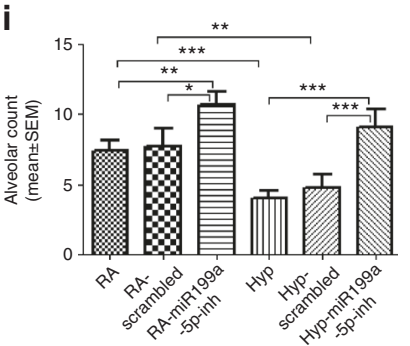

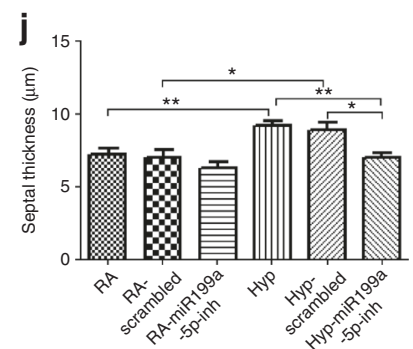
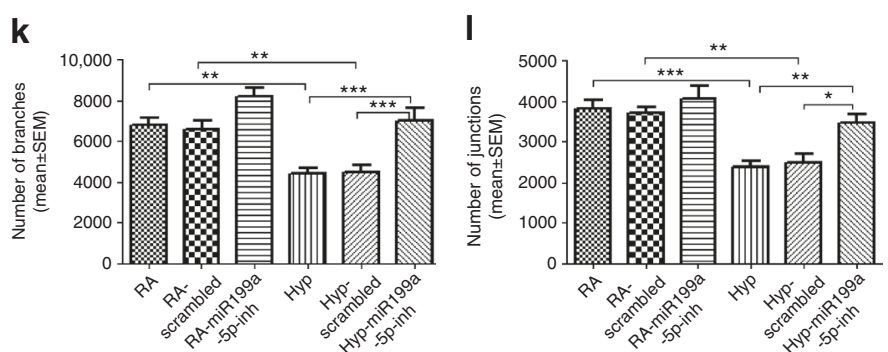

Fig. 8 miR199a-5p-inhibitor improves the lung architecture in hyperoxia (Hyp)-induced acute lung injury (HALI). Representative images of lung histology (hematoxylin and eosin (H\&E) stain) of newborn (NB) wild-type mice treated with miR199a-5p-inhibitor (20 $\mu M$; PN2 and PN4 intranasally), along with appropriate controls, after room air (RA) or Hyp exposure for 7 postnatal (PN) days (a-f). Scale bar: $100 \mu$ m. Morphometry assessed as chord length, alveolar area, alveolar count, septal thickness, and number of branches and junctions ( $\mathbf{g}-\mathbf{I}$ ), as analyzed by the ImageJ software. Values are means \pm SEM of 8-12 animals in each group. ${ }^{*} P<0.05,{ }^{* *} P<0.01$, and ${ }^{* * *} P<0.001$

that increased expression of miR199a-5p attenuated Cav-1 expression in Hyp-exposed cells and NB mice, and was associated with human BPD. Previous studies have shown that increased expression of miR199a-5p led to decreased Cav-1 in CF macrophages and lung fibroblasts. ${ }^{9,10} \mathrm{~A}$ recent study reported that enhanced expression of miR199a-5p led to increased lung fibrosis. $^{10}$ Thus, these findings and our data suggest a potential role of miR199a-5p in lung pathobiology, including neonatal HALI.

We experimentally confirmed the changes in expression of the downstream target of miR199a-5p, Cav-1. Importantly, Zhang et al. ${ }^{9}$ have also established that miR199a-5p represses Cav-1 and the pharmacological (celecoxib) intervention re-establishes the Akt/miR199a-5p/Cav-1 pathway in CF macrophages, and ameliorates lung "hyper-inflammation" in Cftr-deficient mice. ${ }^{9}$ Furthermore, in a BPD mouse model we showed that Cox2 inhibition using celecoxib ameliorates lung alveolarization that had been injured by Hyp and interferon- $\gamma .28$

Prolonged exposure/ventilation with oxygen to the NB lung activates ROS, which causes an increased influx of inflammatory cytokines, neutrophils, and macrophages, ${ }^{2}$ hyperpermeability, coagulopathy, and collagen deposition, as well as impairment of lung alveolarization and vascularization. ${ }^{29,30}$ These features are the hallmark of HALI and BPD. Hyp triggers a cascade of signaling pathways at multiple cellular responses such as apoptosis, necrosis, or repair ${ }^{31,32}$ In line with this, we found that Hyp exposure induces dysregulation of lung architecture, and increased inflammatory cytokines, neutrophils, and macrophages in NB mice.

Additionally, we ${ }^{11}$ have demonstrated that IL- 6 was increased in HALI. Another study showed that lysophosphatidic acid receptor 1 deficiency protected NB rats against LPS-induced lung injury by reducing pulmonary influx of macrophages, neutrophils, and IL-6 production. ${ }^{32}$ Recent studies have shown that TLR4 also plays a significant role in HALI models. These studies have suggested that upregulation of TLR4, IL-6, TNFa, apoptosis, and inflammation by Hyp was ameliorated by vitamin $D$ administration, which antagonized the activation of TLR4 and therefore alleviated inflammation, reduced apoptosis, and preserved lung structure. $^{33,34}$ Adhesion molecules such as E-selectin are crucial for initiating inflammatory responses. The findings observed in the present study suggest that E-selectin contributes to lung inflammation in Hyp. Downregulation of E-selectin expression, with subsequent decrease in neutrophil influx recruitment, may be responsible for reduced inflammation and improved lung architecture. $^{35}$

Akt/protein kinase $B$ is one vital regulatory molecule responsible for maintaining cell viability. ${ }^{36}$ The activation of Akt and post-translational phosphorylation of Akt has been shown to protect a wide range of cell types under stress. To evaluate the possible involvement of pAkt/Akt, we determined the level of lung pAkt/Akt expression in NB HALI model. Interestingly, the expression of post-translational phosphorylation of Akt was decreased in the lungs of animals exposed to Hyp. This observation indicates a potential role of the prosurvival Akt pathway. ${ }^{36}$ Investigators have reported that pharmacologic Akt inhibition impaired alveolarization and produced histologic changes reminiscent of BPD in NB rats. ${ }^{36}$ Likewise, our results indicated that miR199a-5p-mimic (which decreased pAkt level) impaired lung architecture.

Using gain- and loss-of-function strategies (i.e., miR199a-5pmimic and miR199a-5p-inhibitor, respectively), we observed that miR199a-5p-mimic further worsened the HALI phenotype including lung morphometry, along with increased influx of inflammatory cells (neutrophil, macrophages), cytokines (IL-6), and enhanced levels of inflammatory markers (TLR4, E-selectin and IL-6). On the other hand, inhibition of miR199a-5p attenuated the 


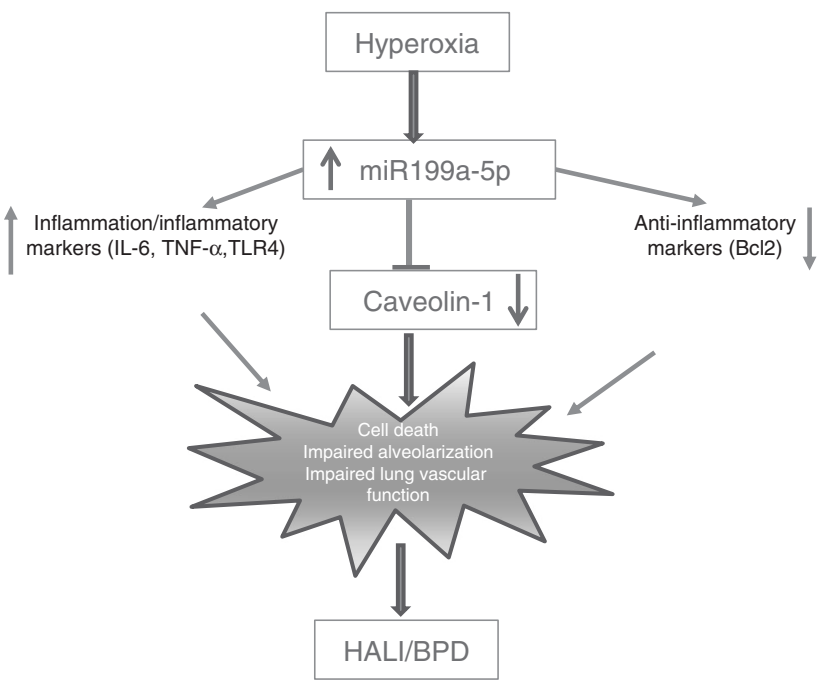

Fig. 9 Proposed schematic representation of the role of miR199a-5p in hyperoxia-induced lung injury/bronchopulmonary dysplasia (HALI/BPD). Hyperoxia exposure to the developing lung results in increased expression of miR199a-5p, which causes decreased levels of caveolin-1, Bcl2 and increased interleukin-6 (IL-6), tumor necrosis factor- $\alpha$ (TNF- $\alpha$ ), toll-like receptor 4 (TLR4) that contribute to increased vascular leak, increased apoptotic cell death, and inflammation, leading to impaired alveolarization and vascular function. This eventually leads to compromised lung physiology that manifests as HALI/BPD.

HALI pulmonary phenotype, suggesting that miR199a-5p-inhibitor could have a potential preventative therapeutic role for HALI and BPD. Furthermore, accumulating evidence suggests that HALI/LPS perpetuates proinflammatory response in the fetal lung, which finally impacts on lung development. ${ }^{8}$ Previous studies from our research laboratory and others also have suggested increased levels of proinflammatory cytokines in HALI- and LPS-exposed NB mice models. ${ }^{8,11,28,37}$ Non-putative targets (TLR4, TNFa, IL-6, and Eselectin) and apoptotic signals were improved, suggesting that miR199a-5p-inhibitor attenuated lung inflammation along with improved alveolarization, alveolar count, chord length, and septal thickness in developing lungs in HALI mice.

Limitation of the study

Our study was carried out to seek answers for acute pathological changes during Hyp under miR199a-5p-mimic and miR199a-5pinhibitor. We studied cell lines and neonatal mice; however, primary mice lung endothelial cells from the experimental mice would have provided results in a cell-specific manner. Thus, further study is warranted to see the long-term effect in mice.

\section{CONCLUSIONS}

Overall, our data suggest that Hyp upregulates mechanisms of cellular injury, which contribute to impaired lung development and function. We observed that overexpression of miR199a-5p leads to repression of transmembrane protein (Cav-1) and enhanced proinflammatory responses and altered lung homeostasis. Conversely, miR199a-5p-inhibitor reverses proinflammatory to anti-inflammatory pathways and attenuates abnormal lung structure. Thus, exploring the contributing mechanisms of miR199a-5p-inhibitor could help identify potential therapeutic strategies to improve lung structure and function of patients with HALI/BPD. A schematic presentation of miR199a-5p's role in HAL/ BPD is shown in Fig. 9.

\section{ACKNOWLEDGEMENTS}

The work was supported by Departmental Funds (V.B.)

\section{AUTHOR CONTRIBUTIONS}

Concept and design: M.A.A., V.B. Acquisition of data: M.A.A., S.G.B., Z.H.A., V.B. Data analysis and interpretation: M.A.A., V.B. Drafting and/or critical revision for intellectual content: M.A.A., V.B. All authors have approved the version of the submitted manuscript.

\section{ADDITIONAL INFORMATION}

The online version of this article (https://doi.org/10.1038/s41390-019-0524-3) contains supplementary material, which is available to authorized users.

Competing interests: The authors declare no competing interests.

Publisher's note: Springer Nature remains neutral with regard to jurisdictional claims in published maps and institutional affiliations.

\section{REFERENCES}

1. Galambos, C. \& Demello, D. E. Regulation of alveologenesis: clinical implications of impaired growth. Pathology 40, 124-140 (2008).

2. Sureshbabu, A. et al. Inhibition of regulatory-associated protein of mechanistic target of rapamycin prevents hyperoxia-induced lung injury by enhancing autophagy and reducing apoptosis in neonatal mice. Am. J. Respir. Cell Mol. Biol. 55, 722-735 (2016)

3. Bhandari, A. \& Bhandari, V. Pitfalls, problems, and progress in bronchopulmonary dysplasia. Pediatrics 123, 1562-1573 (2009).

4. Bhandari, V. Hyperoxia-derived lung damage in preterm infants. Semin. Fetal Neonatal Med. 15, 223-229 (2010).

5. Bhandari, A. \& Bhandari, V. "New" bronchopulmonary dysplasia-a clinical review. Clin. Pulm. Med. 18, 137-143 (2011).

6. Shukla, G. C., Singh, J. \& Barik, S. MicroRNAs: processing, maturation, target recognition and regulatory functions. Mol. Cell. Pharmcol. 3, 83-92 (2011).

7. Syed, M. et al. Hyperoxia causes miR-34a-mediated injury via angiopoietin-1 in neonatal lungs. Nat. Commun. 8, 1173 (2017).

8. Durrani-Kolarik, S. et al. miR-29b supplementation decreases expression of matrix proteins and improves alveolarization in mice exposed to maternal inflammation and neonatal hyperoxia. Am. J. Physiol. Lung Cell. Mol. Physiol. 313, L339-L349 (2017).

9. Zhang, P. X. et al. Pharmacological modulation of the AKT/microRNA-199a-5p/ CAV1 pathway ameliorates cystic fibrosis lung hyper-inflammation. Nat. Commun. 6, 6221 (2015).

10. Lino Cardenas, C. L. et al. miR-199a-5p Is upregulated during fibrogenic response to tissue injury and mediates TGFbeta-induced lung fibroblast activation by targeting caveolin-1. PLoS Genet. 9, e1003291 (2013).

11. Choo-Wing, R., Nedrelow, J. H., Homer, R. J., Elias, J. A. \& Bhandari, V. Developmental differences in the responses of IL- 6 and IL-13 transgenic mice exposed to hyperoxia. Am. J. Physiol. Lung Cell. Mol. Physiol. 293, L142-150 (2007).

12. Syed, M. A. et al. Expression of TREM-1 is inhibited by PGD2 and PGJ2 in macrophages. Exp. Cell Res. 316, 3140-3149 (2010).

13. Syed, M. A. \& Bhandari, V. Hyperoxia exacerbates postnatal inflammation-induced lung injury in neonatal BRP-39 null mutant mice promoting the M1 macrophage phenotype. Mediat. Inflamm. 2013, 457189 (2013).

14. Sureshbabu, A. et al. Conditional overexpression of TGFbeta1 promotes pulmonary inflammation, apoptosis and mortality via TGFbetaR2 in the developing mouse lung. Respir. Res. 16, 4 (2015).

15. Li, Z. et al. A potential role of the JNK pathway in hyperoxia-induced cell death, myofibroblast transdifferentiation and TGF-beta1-mediated injury in the developing murine lung. BMC Cell Biol. 12, 54 (2011).

16. Harijith, A. et al. A role for matrix metalloproteinase 9 in IFNgamma-mediated injury in developing lungs: relevance to bronchopulmonary dysplasia. Am. J. Respir. Cell Mol. Biol. 44, 621-630 (2011).

17. Chaubey, S. et al. Early gestational mesenchymal stem cell secretome attenuates experimental bronchopulmonary dysplasia in part via exosome-associated factor TSG-6. Stem Cell Res. Ther. 9, 173 (2018).

18. Mizuno, S. et al. MicroRNA-199a-5p is associated with hypoxia-inducible factor1alpha expression in lungs from patients with COPD. Chest 142, 663-672 (2012).

19. Hassan, T. et al. miR-199a-5p silencing regulates the unfolded protein response in chronic obstructive pulmonary disease and alpha1-antitrypsin deficiency. Am. J. Respir. Crit. Care Med. 189, 263-273 (2014). 
Hyperoxia causes miR199a-5p-mediated injury in the developing lung

MA Alam et al.

588

20. Osei, E. T. et al. Unravelling the complexity of COPD by microRNAs: it's a small world after all. Eur. Respir. J. 46, 807-818 (2015).

21. Zhang, X. et al. MicroRNA expression profile in hyperoxia-exposed newborn mice during the development of bronchopulmonary dysplasia. Respir. Care 56, 1009-1015 (2011).

22. Narasaraju, T. et al. Role of microRNA-150 and glycoprotein nonmetastatic melanoma protein $B$ in angiogenesis during hyperoxia-induced neonatal lung injury. Am. J. Respir. Cell Mol. Biol. 52, 253-261 (2015).

23. Benlhabib, H., Guo, W., Pierce, B. M. \& Mendelson, C. R. The miR-200 family and its targets regulate type II cell differentiation in human fetal lung. J. Biol. Chem. 290, 22409-22422 (2015)

24. Olave, N. et al. Regulation of alveolar septation by microRNA-489. Am. J. Physiol. Lung Cell. Mol. Physiol. 310, L476-487 (2016).

25. Go, H. et al. MiR-196a regulates heme oxygenase- 1 by silencing Bach 1 in the neonatal mouse lung. Am. J. Physiol. Lung Cell. Mol. Physiol. 311, L400-411 (2016).

26. Jin, Y., Lee, S. J., Minshall, R. D. \& Choi, A. M. Caveolin-1: a critical regulator of lung injury. Am. J. Physiol. Lung Cell. Mol. Physiol. 300, L151-160 (2011).

27. Wang, X. M. et al. Caveolin-1: a critical regulator of lung fibrosis in idiopathic pulmonary fibrosis. J. Exp. Med. 203, 2895-2906 (2006).

28. Choo-Wing, R. et al. Hyperoxia and interferon-gamma-induced injury in developing lungs occur via cyclooxygenase-2 and the endoplasmic reticulum stress-dependent pathway. Am. J. Respir. Cell. Mol. Biol. 48, 749-757 (2013).
29. Mach, W. J., Thimmesch, A. R., Pierce, J. T. \& Pierce, J. D. Consequences of hyperoxia and the toxicity of oxygen in the lung. Nurs. Res. Pract. 2011, 260482 (2011).

30. Maniatis, N. A. et al. Role of caveolin-1 expression in the pathogenesis of pulmonary edema in ventilator-induced lung injury. Pulm. Circ. 2, 452-460 (2012).

31. Perrone, S., Bracciali, C., Di Virgilio, N. \& Buonocore, G. Oxygen use in neonatal care: a two-edged sword. Front. Pediatr. 4, 143 (2016).

32. Chen, X. et al. Adult lysophosphatidic acid receptor 1-deficient rats with hyperoxia-induced neonatal chronic lung disease are protected against lipopolysaccharide-induced acute lung injury. Front. Physiol. 8, 155 (2017).

33. Yao, L. et al. Vitamin D attenuates hyperoxia-induced lung injury through downregulation of Toll-like receptor 4. Int. J. Mol. Med. 39, 1403-1408 (2017).

34. Chen, Y. et al. Attenuation of hyperoxia-induced lung injury in neonatal rats by 1alpha,25-dihydroxyvitamin D3. Exp. Lung Res. 41, 344-352 (2015).

35. Ramsay, P. L., Geske, R. S., Montgomery, C. A. \& Welty, S. E. Increased soluble Eselectin is associated with lung inflammation, and lung injury in hyperoxiaexposed rats. Toxicol. Lett. 87, 157-165 (1996).

36. Alphonse, R. S. et al. Activation of Akt protects alveoli from neonatal oxygeninduced lung injury. Am. J. Respir. Cell. Mol. Biol. 44, 146-154 (2011).

37. Balany, J. \& Bhandari, V. Understanding the impact of infection, inflammation, and their persistence in the pathogenesis of bronchopulmonary dysplasia. Front. Med. (Lausanne) 2, 90 (2015). 